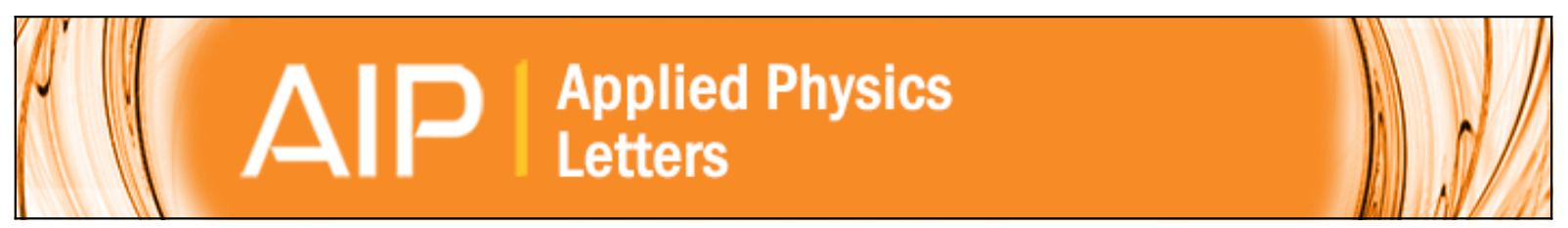

\title{
Direct fiber-coupled single photon source based on a photonic crystal waveguide
}

Byeong-Hyeon Ahn, Chang-Min Lee, Hee-Jin Lim, Thomas W. Schlereth, Martin Kamp, Sven Höfling, and YongHee Lee

Citation: Applied Physics Letters 107, 081113 (2015); doi: 10.1063/1.4929838

View online: http://dx.doi.org/10.1063/1.4929838

View Table of Contents: http://scitation.aip.org/content/aip/journal/apl/107/8?ver=pdfcov

Published by the AIP Publishing

\section{Articles you may be interested in}

Cross two photon absorption in a silicon photonic crystal waveguide fiber taper coupler with a physical junction J. Appl. Phys. 117, 033101 (2015); 10.1063/1.4905615

Efficient coupling of single photons to ridge-waveguide photonic integrated circuits Appl. Phys. Lett. 102, 131105 (2013); 10.1063/1.4799669

Enhanced spontaneous emission from quantum dots in short photonic crystal waveguides Appl. Phys. Lett. 100, 061122 (2012); 10.1063/1.3683541

Efficient quantum dot single photon extraction into an optical fiber using a nanophotonic directional coupler Appl. Phys. Lett. 99, 121101 (2011); 10.1063/1.3617472

Probing the dispersive and spatial properties of photonic crystal waveguides via highly efficient coupling from fiber tapers

Appl. Phys. Lett. 85, 4 (2004); 10.1063/1.1767954

\section{AlP $\mid$ APL Photonics}

APL Photonics is pleased to announce Benjamin Eggleton as its Editor-in-Chief

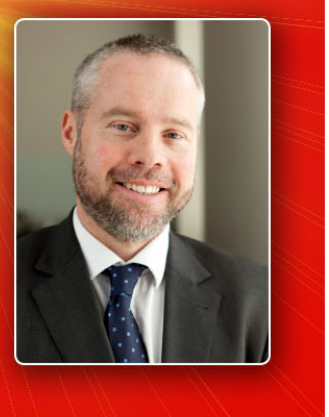




\title{
Direct fiber-coupled single photon source based on a photonic crystal waveguide
}

\author{
Byeong-Hyeon Ahn, ${ }^{1, a)}$ Chang-Min Lee, ${ }^{1}$ Hee-Jin Lim, ${ }^{1}$ Thomas W. Schlereth, ${ }^{2}$ \\ Martin Kamp, ${ }^{2}$ Sven Höfling, ${ }^{2,3}$ and Yong-Hee Lee ${ }^{1,4}$ \\ ${ }^{1}$ Department of Physics, KAIST, Daejeon 305-701, South Korea \\ ${ }^{2}$ Technische Physik, Physikalisches Institut and Wilhelm Conrad Röntgen-Center for Complex Material \\ Systems, Universität Würzburg, Am Hubland, D-97074 Würzburg, Germany \\ ${ }^{3}$ SUPA, School of Physics and Astronomy, University of St. Andrews, St. Andrews KY16 9SS, United Kingdom \\ ${ }^{4}$ Graduate School of Nanoscience and Technology (WCU), KAIST, Daejeon 305-701, South Korea
}

(Received 13 May 2015; accepted 19 August 2015; published online 28 August 2015)

\begin{abstract}
A single photon source plays a key role in quantum applications such as quantum computers and quantum communications. Epitaxially grown quantum dots are one of the promising platforms to implement a good single photon source. However, it is challenging to realize an efficient single photon source based on semiconductor materials due to their high refractive index. Here we demonstrate a direct fiber coupled single photon source with high collection efficiency by employing a photonic crystal $(\mathrm{PhC})$ waveguide and a tapered micro-fiber. To confirm the single photon nature, the second-order correlation function $\mathrm{g}^{(2)}(\tau)$ is measured with a Hanbury BrownTwiss setup. The measured $\mathrm{g}^{(2)}(0)$ value is 0.15 , and we can estimate $24 \%$ direct collection efficiency from a quantum dot to the fiber. (C) 2015 AIP Publishing LLC.

[http://dx.doi.org/10.1063/1.4929838]
\end{abstract}

The field of quantum information processing has been growing rapidly over the last two decades. Unique properties of quantum states allow us to develop new applications such as quantum computers and quantum communications. ${ }^{1-3}$ Several experiments have shown possibilities of implementation of quantum applications based on a single atom ${ }^{4,5}$ or a single ion. ${ }^{6-8}$ However, the scalability has been a big practical issue in these schemes. Semiconductor technologies using quantum dots (QDs) have been spotlighted as one of alternative ways to resolve it. ${ }^{9}$ Thanks to the modern epitaxial growth and nano-fabrication capabilities, quantum experiments in small scale become possible. ${ }^{10,11}$ However, the high refractive index of semiconductor materials hinders efficient extraction of photons, which degrades the quality of quantum states. Thus, it is essential to collect photons from a semiconductor-based emitter with high efficiency.

Single photons from a quantum dot have shown clear quantum features. ${ }^{9,12,13}$ Recently, various types of single photon sources have been demonstrated with photonic crystals, micropillars, nanotrumpets, and photonic nanowires, ${ }^{13-19}$ and many groups are in efforts to realize single photon sources with high quantum efficiency, high photon extraction efficiency, high single photon purity, and fast repetition rate. ${ }^{13-15,18-22}$ However, it is very challenging to satisfy all of these properties simultaneously. Micropillars and photonic nanowires show better collection with high numerical aperture objective lenses due to their vertical structure. However, planar type sources like photonic crystals are also important to chip scale integration applications. In this study, we demonstrate a single photon source with high extraction efficiency based on a PhC waveguide by coupling to a

\footnotetext{
a) Present address: Department of Electrical and Computer Engineering, Duke University, Durham, North Carolina 27708, USA. Electronic mail: seygene@kaist.ac.kr
}

tapered micro-fiber. Though photons cannot escape easily from a high refractive material because of the total internal reflection, the perfect phase matching can improve the extraction efficiency dramatically. Direct collection of single photons with a tapered micro-fiber enables us to transfer them directly to a distance without going out of the fiber.

A photonic crystal $(\mathrm{PhC})$ waveguide is a $\mathrm{PhC}$ pattern with one line of filled air holes as shown in Fig. 1(a). ${ }^{23}$ Dispersion characteristics of a PhC guided mode are summarized in Fig. 1(b). ${ }^{24,25}$ There are two guided modes whose $E_{y}$ fields show different symmetries. The even guided mode has an electric field maximum at the center of waveguide, which encourages photons from quantum dots to couple into the waveguide mode efficiently. However, these guided modes are not allowed to couple with the free space by definition. In this study, we employ a tapered micro-fiber that has a diameter comparable to the waveguide width in order to funnel photons from a $\mathrm{PhC}$ waveguide. In the dispersion curve (Fig. 1(b)), one can find a cross point of phase matching between two k-vectors of the even $\mathrm{PhC}$ guided mode and the fiber guided mode. Once the phase matching condition is satisfied, photons in waveguide can couple seamlessly to the micro-fiber. ${ }^{26}$

In the experimental setup shown in Fig. 2(a), a 780-nm femto-second laser is fed through one end of the fiber to generate excitons in quantum dots. The signal from quantum dots is collected at the other end of the same fiber. It is delivered to a spectrometer through lens and mirrors. A Si array detector is used to record QD spectra, and two avalanche photodiodes (APDs) and a time amplitude converter are used for the second correlation measurement. To move the fiber close to the intended position, low magnification imaging was used first. Then, the lens was changed to a $50 \times$ magnification objective lens. The contact point of curved tapered micro-fiber is shown in Fig. 2, and the position of the fiber 
(a)

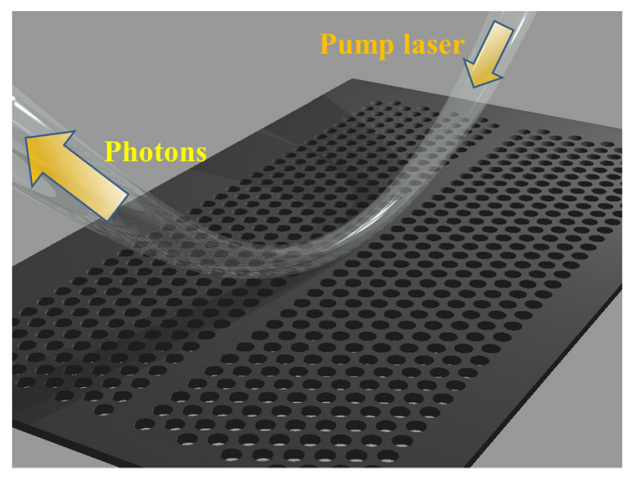

(b)

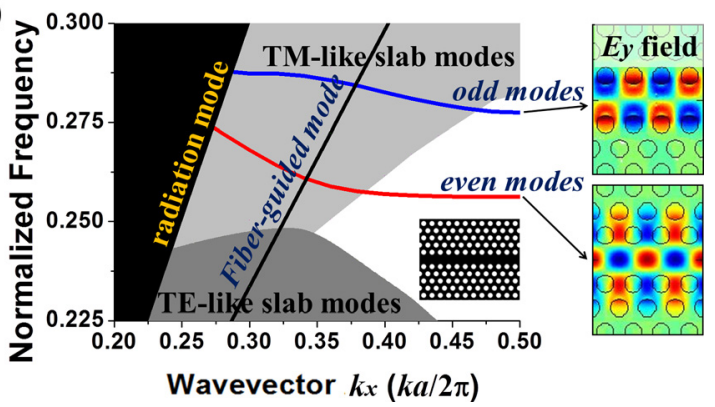

FIG. 1. Photonic crystal waveguide. (a) Schematic diagram of integrated system of photonic crystal waveguide and a tapered-fiber. (b) Dispersion of photonic crystal waveguide. Blue line shows odd modes, and red line shows even modes in photonic crystal waveguide. Field distributions of even and odd modes are at $\mathrm{k}=0.5$.

was controlled by piezoelectric motor stages. A photonic crystal waveguide was fabricated with 170 -nm-thick GaAs slab containing a few InAs quantum dots per square micron, emitting near $950 \mathrm{~nm}$ in wavelength (Fig. 2(b)).

The tapered micro-fiber was fabricated using heat-andpull techniques with a conventional single mode optical fiber having $125-\mu \mathrm{m}$ cladding. ${ }^{27,28}$ Fiber tapering was performed by heating an optical fiber with a small oscillating flame and simultaneously pulling the fiber in opposite directions by motorized translational stages. $1550-\mathrm{nm}$ probe light was inserted and monitored during the pulling process to check the single mode condition of the air-cladding mode, which shows up when the diameter is $\sim 1.0 \mu \mathrm{m}$. The probe light transmission through the tapered region was over $80 \%$. The tapered micro-fiber was then curved to a loop whose radius of curvature is $100-150 \mu \mathrm{m}$. The curved tapered micro-fiber was fixed to a small aluminum unit by epoxy.

Theoretically, the guided mode of an infinite waveguide should be continuous. However, the finite $\mathrm{PhC}$ waveguide causes the mode to be discrete as shown in Fig. 2(c). Measured data are compared with those of 3D finitedifference time-domain (FDTD) computations in Fig. 3(a) where the vertical scale is expressed in photon energy. The length of measured waveguide is $41 a(\sim 10 \mu \mathrm{m})$, where $a$ is the lattice constant of the $\mathrm{PhC}$ waveguide. The dispersion curve is divided by 42 (41a-long waveguide acts as effectively $42 a$-long waveguide ${ }^{29}$ ). The measured peaks in the spectrum agree well with the predicted points on the dispersion curve. This spectrum was taken with sufficiently strong pump power to expose all the discrete and weak $\mathrm{PhC}$ guided modes. Under weak pump conditions, those peaks near the $\mathrm{k}$-vector matching point are relatively

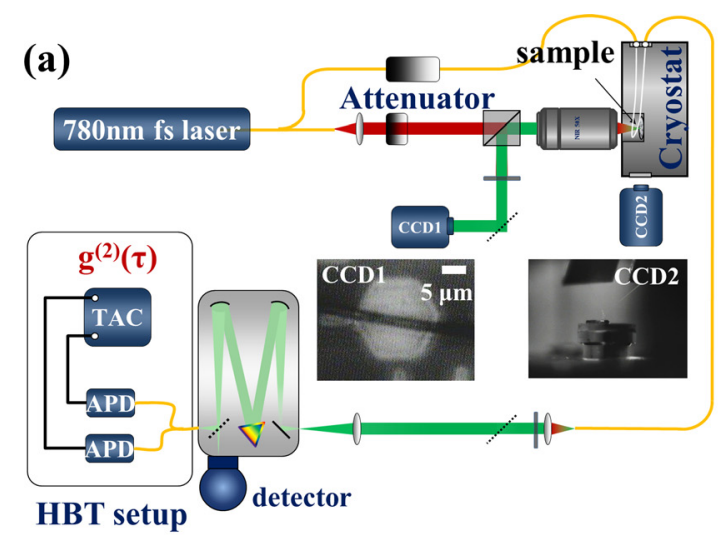

(b)
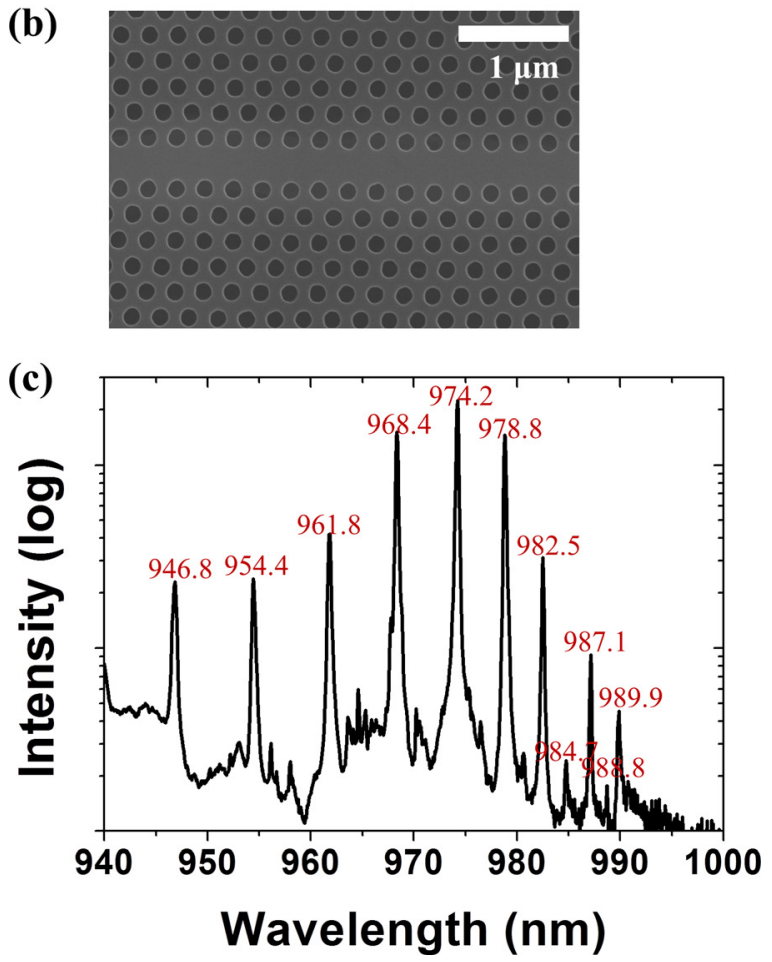

FIG. 2. Measurement. (a) An experimental measurement setup. CCD1 is monitoring the fiber and $\mathrm{PhC}$ waveguide on top and CCD2 is monitoring them by side. (b) SEM image of fabricated sample of $\mathrm{PhC}$ waveguide containing a few QDs. (c) Measurement of photonic crystal waveguide modes through the tapered micro-fiber.

brighter than other peaks. Electric field intensity distributions of FDTD calculations show very different behaviors at two coupling points designated as b and c in Fig. 3(a). When the phase matching condition is satisfied (point b), light in the $\mathrm{PhC}$ waveguide funnels smoothly into the fiber (Fig. 3(b)). In comparison at point c, photons in the PhC waveguide are unable to escape (Fig. 3(c)). This observation agrees well with that of the measurement as shown in Fig. 3(a).

A spectrum with pump power of $45 \mathrm{nW}$ is shown in Fig. 4(a). Among several peaks the 978 -nm peak is the strongest. The strength of this peak was measured as a function of the pump power as shown in Fig. 4(b). It shows linear dependence in the weak pump regime and becomes saturated in the strong pump regime. The inset of Fig. 4(b) is a reproduction in linear scale in both axes. This saturation behavior is typical of a single QD emitter. We measured the second-order correlation to confirm the single photon 
(a)

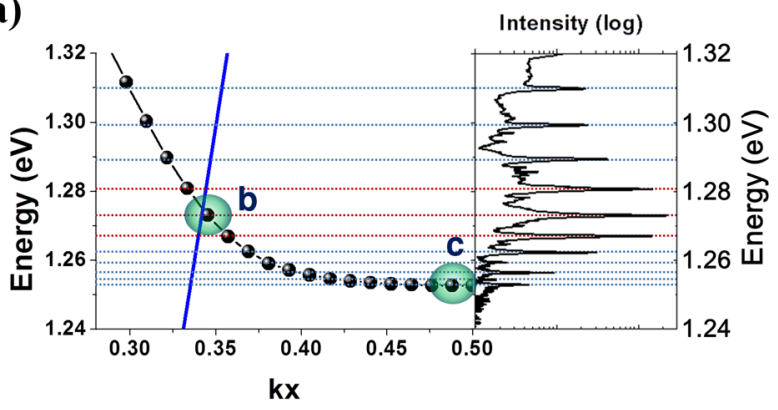

(b)

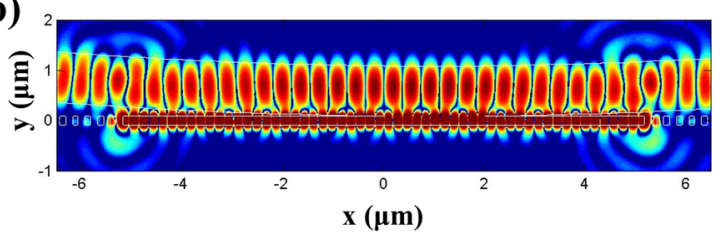

(c)

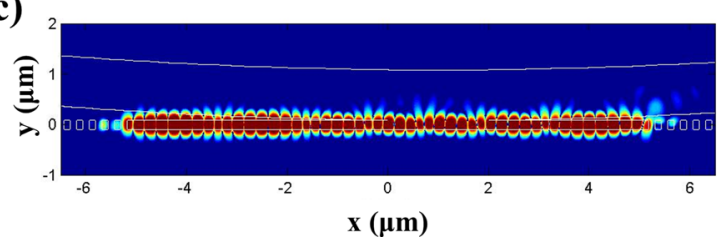

FIG. 3. Coupling efficiency to the tapered micro-fiber. (a) Comparison of dispersion and measurement of $\mathrm{PhC}$ waveguide. (b) Electric field intensity (log scale) in XZ plane of the mode near tapered-fiber guided line (point b). (c) Electric field intensity (log scale) in XZ plane of the mode near the edge (point c).

characteristics with a Hanbury Brown-Twiss setup. Measured results are shown in Figs. 4(c) and 4(d) after 2-h integration. $\mathrm{g}^{(2)}(0)=0.15$ and $\mathrm{g}^{(2)}(0)=0.30$ were obtained with pump powers of $26 \mathrm{nW}$ and $700 \mathrm{nW}$, respectively. In this measurement, two photon emissions and correlation with dark counts of the detectors were also included in addition to the single photons. We would like to emphasize that the generated single photons remain in the fiber all the time.

To estimate the collection efficiency, we measured transmissions of all optics in the setup (Fig. 2(a)). The total transmission was $3.1 \%$ including those from the tapered micro-fiber section (0.69), the transmission of lenses and spectrometer (0.42), the splitting ratio of fiber splitter (47:53), and the detection efficiency of APD at $978 \mathrm{~nm}$ (0.20). Based on the count rate in APD and the corrected photon count rate by the factor of $\left[1-\mathrm{g}^{(2)}(0)\right]^{1 / 2}$, 22 we estimate that $1.9 \times 10^{7}$ photons/s was coupled to the fiber at the saturated pump power. The repetition rate of the pump laser was $8.0 \times 10^{7} / \mathrm{s}$. Therefore, the collection efficiency to the fiber is estimated to be $24 \%$, which includes internal quantum efficiency, spontaneous emission factor $\beta$, and output coupling efficiency $\eta$. The internal quantum efficiency is typically over $0.90,{ }^{22}$ and the spontaneous emission factor of a similar structure is around $0.80 .^{30,31}$ Thus, we can roughly estimate the coupling efficiency $\eta$ between $\mathrm{PhC}$ waveguide and the tapered micro-fiber is around $33 \%$. This value of coupling efficiency compares well with the other group's results. ${ }^{15-17,32,33}$ Theoretically, the coupling efficiency can be over $90 \%$ depending on the slab thickness. ${ }^{25}$ The thinner slab allows the better coupling, but the thin slab causes fabrication difficulty. The discrepancy between the calculation and the measurement estimation is due to the fabrication imperfection of $\mathrm{PhC}$ and the tapered micro-fiber.

In conclusion, we demonstrated an efficient single photon source combined with a tapered micro-fiber and confirmed it by measuring the second order correlation of the signal. The collection efficiency of photons was estimated to $24 \%$. The proposed scheme here can be applied to quantum communication applications since single photons exist inside of the fiber from the birth. In addition, photons can be delivered to anywhere with very small losses, which is advantageous in various quantum applications using light quanta. (a)

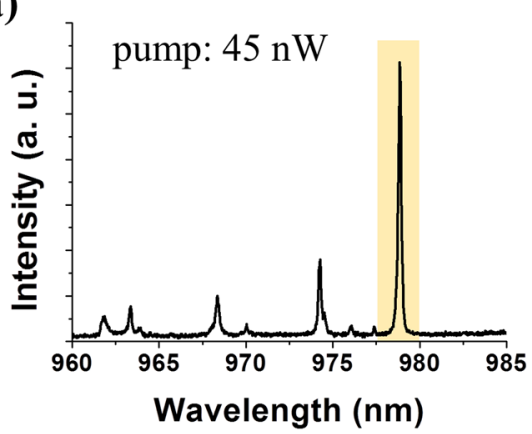

(c)

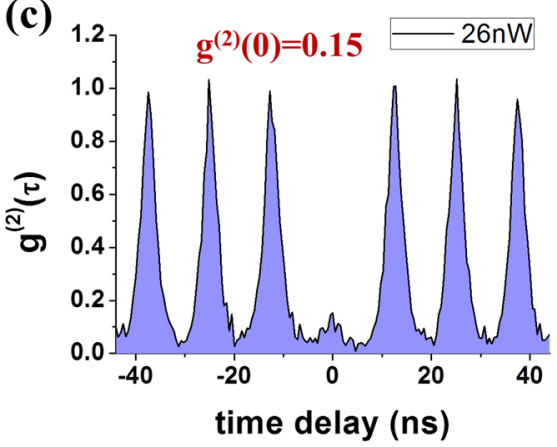

(b)

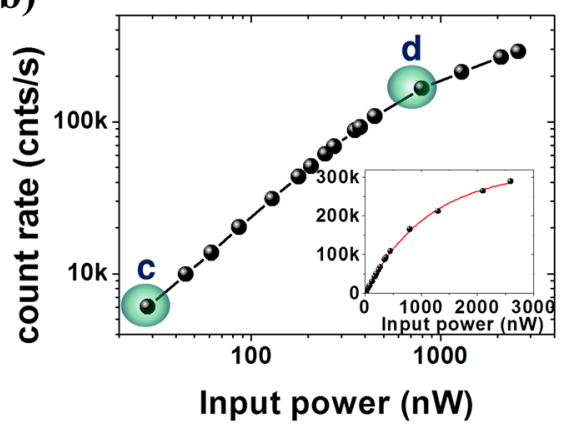

(d)

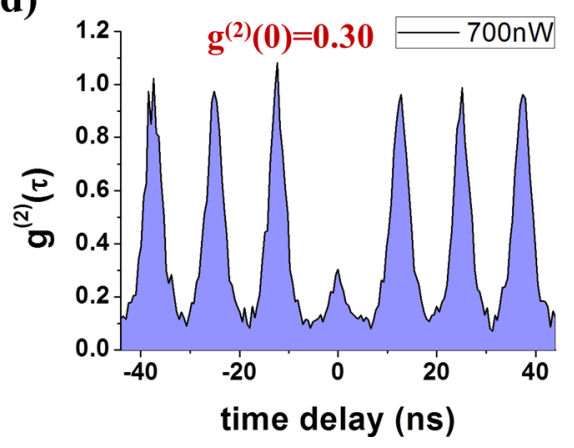

FIG. 4. Spectrum and $\mathrm{g}^{(2)}(\tau)$ correlation. (a) Measured spectra with $45 \mathrm{nW}$ of pump power. (b) Pump power dependence (log-log scale) of the peak $978.8 \mathrm{~nm}$. Inset shows linear-linear power dependence. (c) and (d) Measured $\mathrm{g}^{(2)}(\tau)$ correlation at $26 \mathrm{nW}$ and $700 \mathrm{nW}$ pump power. 
This research was supported by National Research Foundation of Korea (NRF) funded by Korea Government (MSIP) (Nos. 20070093863 and 2014M3C1A3052537). B.H. Ahn acknowledges the support from National Junior Research Fellowship (No. 20110031262).

${ }^{1}$ R. P. Feynman, Found. Phys. 16, 507 (1986).

${ }^{2}$ T. D. Ladd, F. Jelezko, R. Laflamme, Y. Nakamura, C. Monroe, and J. L. O. R. Brien, Nature 464, 45 (2010).

${ }^{3}$ N. Gisin, G. Ribordy, W. Tittel, and H. Zbinden, Rev. Mod. Phys. 74, 145 (2002).

${ }^{4}$ S. Ritter, C. Nölleke, C. Hahn, A. Reiserer, A. Neuzner, M. Uphoff, M. Mücke, E. Figueroa, J. Bochmann, and G. Rempe, Nature 484, 195 (2012).

${ }^{5}$ H. P. Specht, C. Nölleke, A. Reiserer, M. Uphoff, E. Figueroa, S. Ritter, and G. Rempe, Nature 473, 190 (2011).

${ }^{6}$ H. Häffner, C. F. Roos, and R. Blatt, Phys. Rep. 469, 155 (2008).

${ }^{7}$ R. Blatt and D. Wineland, Nature 453, 1008 (2008).

${ }^{8}$ L. M. Duan and C. Monroe, Rev. Mod. Phys. 82, 1209 (2010).

${ }^{9}$ A. J. Shields, Nat. Photonics 1, 215 (2007).

${ }^{10}$ H. Mabuchi and A. C. Doherty, Science 298, 1372 (2002).

${ }^{11}$ D. Englund, A. Faraon, I. Fushman, N. Stoltz, P. Petroff, and J. Vučković, Nature 450, 857 (2007).

${ }^{12}$ S. Buckley, K. Rivoire, and J. Vučković, Rep. Prog. Phys. 75, 126503 (2012).

${ }^{13}$ J. Claudon, J. Bleuse, N. S. Malik, M. Bazin, P. Jaffrennou, N. Gregersen, C. Sauvan, P. Lalanne, and J. M. Gérard, Nat. Photonics 4, 174 (2010).

${ }^{14}$ O. Gazzano, S. Michaelis de Vasconcellos, C. Arnold, A. Nowak, E. Galopin, I. Sagnes, L. Lanco, A. Lemaître, and P. Senellart, Nat. Commun. 4, 1425 (2013).

${ }^{15}$ M. Toishi, D. Englund, A. Faraon, and J. Vuckovic, Opt. Express 17, 14618 (2009)

${ }^{16}$ T. Heindel, C. Schneider, M. Lermer, S. H. Kwon, T. Braun, S. Reitzenstein, S. Höfling, M. Kamp, and A. Forchel, Appl. Phys. Lett. 96, 011107 (2010).
${ }^{17}$ M. Davanço, M. T. Rakher, W. Wegscheider, D. Schuh, A. Badolato, and K. Srinivasan, Appl. Phys. Lett. 99, 121101 (2011).

${ }^{18}$ M. Munsch, N. S. Malik, E. Dupuy, A. Delga, J. Bleuse, J.-M. Gérard, J. Claudon, N. Gregersen, and J. Mork, Phys. Rev. Lett. 110, 177402 (2013).

${ }^{19}$ M. E. Reimer, G. Bulgarini, N. Akopian, M. Hocevar, M. B. Bavinck, M. A. Verheijen, E. P. A. M. Bakkers, L. P. Kouwenhoven, and V. Zwiller, Nat. Commun. 3, 737 (2012).

${ }^{20}$ S. Strauf, N. G. Stoltz, M. T. Rakher, L. A. Coldren, P. M. Petroff, and D. Bouwmeester, Nat. Photonics 1, 704 (2007).

${ }^{21}$ W. L. Barnes, G. Bjork, J. M. Gérard, P. Jonsson, J. Wasey, P. T. Worthing, and V. Zwiller, Eur. Phys. J. D 18, 197 (2002).

${ }^{22}$ M. Pelton, C. Santori, J. Vučković, B. Zhang, G. S. Solomon, J. Plant, and Y. Yamamoto, Phys. Rev. Lett. 89, 233602 (2002).

${ }^{23}$ M. Notomi, A. Shinya, K. Yamada, J. I. Takahashi, C. Takahashi, and I. Yokohama, IEEE J. Quantum Electron. 38, 736 (2002).

${ }^{24}$ M. K. Kim, J. Y. Kim, J. H. Kang, B.-H. Ahn, and Y. H. Lee, Laser Photonics Rev. 5, 479 (2011).

${ }^{25}$ I. Karnadi, J. Y. Kim, B.-H. Ahn, H. J. Lim, and Y. H. Lee, J. Opt. Soc. Am. B 29, 2669 (2012).

${ }^{26}$ P. E. Barclay, K. Srinivasan, M. Borselli, and O. Painter, Electron. Lett. 39, 842 (2003).

${ }^{27}$ M.-K. Kim, I.-K. Hwang, S.-H. Kim, H.-J. Chang, and Y.-H. Lee, Appl. Phys. Lett. 90, 161118 (2007).

${ }^{28}$ C. Grillet, C. Monat, C. Smith, B. J. Eggleton, D. D. Moss, S. Frédérick, D. Dalacu, P. Poole, J. Lapointe, and G. Aers, Opt. Express 15, 1267 (2007).

${ }^{29}$ S. H. Kim, G. H. Kim, S. K. Kim, H. G. Park, Y. H. Lee, and S. B. Kim, J. Appl. Phys. 95, 411 (2004).

${ }^{30}$ T. B. Hoang, J. Beetz, M. Lermer, L. Midolo, M. Kamp, S. Höfling, and A. Fiore, Opt. Express 20, 21758 (2012).

${ }^{31}$ A. Laucht, S. Pütz, T. Günthner, N. Hauke, R. Saive, S. Frédérick, M. Bichler, M. C. Amann, A. Holleitner, M. Kaniber, and J. Finley, Phys. Rev. X 2, 011014 (2012).

${ }^{32}$ X. Liu, T. Asano, S. Odashima, H. Nakajima, H. Kumano, and I. Suemune, Appl. Phys. Lett. 102, 131114 (2013).

${ }^{33}$ A. Schwagmann, S. Kalliakos, I. Farrer, J. P. Griffiths, G. A. C. Jones, D. A. Ritchie, and A. J. Shields, Appl. Phys. Lett. 99, 261108 (2011). 